\title{
Study of Noise in a Small Spanish Town
}

\author{
J.M. Barrigón Morillas, V. Gómez Escobar, J.A. Méndez Sierra and R. Vílchez Gómez \\ Departamento de Física. E. Politécnica, Universidad de Extremadura, \\ Avda. de la Universidad s/n. 10071 Cáceres, Spain
}

(Received 29 June 2001; accepted 14 May 2002)

\begin{abstract}
A study is presented of noise in Navalmoral de la Mata, a small town of Extremadura, Spain. The town was divided into a $200 \mathrm{~m}$ square grid and noise levels were measured at the nodes of this grid. The study was performed in the two months of August and September 1999 in order to compare a vacation and a non-vacation period. Unlike other studies, no major differences were found. The predictive capacity of the measurement model by grid was analysed by comparing the simple grid map (taking the value for a square to be the average of the values at the corners) and the isoline map obtained by a standard interpolation routine with test measurements at points within some of the squares. Agreement was poor, and the isoline map gave only a slight improvement. Even when the size of a part of the grid was reduced to $100 \mathrm{~m}$, the predictive capacity remained low, even though this involved a fourfold increase in time and resources. The noise levels here were lower than in other Spanish towns of similar size but in more developed parts of the country, reflecting the influence of a region's socio-economic situation.
\end{abstract}

${ }^{\dagger}$ Member of the International Institute of Acoustics and Vibration (IIAV)

\section{INTRODUCTION}

The rapid evolution of our society has mainly been driven by great scientific advances. These have brought about important changes, not only technological, but also social and economical. However, these changes have not taken place uniformly in all countries, nor in the different regions within the countries. One such change has been a major growth in population, industry, trade, travel, etc. Perhaps the least desirable consequence of this situation is the current level of pollution of our environment and the associated loss of quality of life.

Acoustical pollution is one part of this problem, and a number of studies have been carried out in the last few decades to characterize the noise levels that citizens are subjected to in their work and daily lives. ${ }^{1-3}$

Extremadura is a Spanish region whose economic indicators show a lower level of development than not only the European average but also the Spanish average. Nevertheless, studies performed in the largest cities of this region ${ }^{4-5}$ found that the acoustical pollution levels are similar to those of other Spanish cities with a similar or larger size, independently of the level of development. ${ }^{6-10}$ Unfortunately, there have been no studies of urban noise in other small or medium sized towns of this region.

In the present work an acoustical study was performed in a small town of Extremadura. This town, Navalmoral de la Mata, has grown steadily in population from the early years of the last century. It now has approximately 16,000 inhabitants. Its main economic activities are in the service sector (with $40 \%$ of the working population) and the industrial sector (with $27 \%$ ). The relative importance of the service sector is because the town is the centre of a flourishing agricultural area and is well communicated with both the surrounding area and the rest of Spain.

Traffic, generally present in all parts of the town, was taken to be the main noise source, since this is usually the most important and widespread noise source in urban areas. $^{4,11}$ To analyse the contribution of traffic noise, daytime noise maps were drawn up. In an attempt to observe the possible differences between vacation and non-vacation periods in this type of town, separate maps were created with data taken in August (the vacation month for most Spaniards) and in September and October. We also studied the relationship between the grid resolution and the quality of the resulting noise map.

\section{METHODS}

In the first stage, the 24-h evolution of $L_{A_{e q}}$ (the energyaverage sound level) was measured in order to have an overall picture of the noise levels and hence to divide the day into time intervals that presented a certain temporal uniformity in this noise index. The sound level meter was placed on the balcony of a typical street, on the fourth floor. Two series of measurements were made. In the first (19-23 July, 1999), a type II sound level meter, Rion NL-04, was used. Noise levels were logged every fifteen minutes. In the second (2-6 August, 1999) a type I sound level meter, Brüel \& Kjær (B\&K) 2236, was used. In this case, noise levels were logged every minute. The data were then used to divide the day into almost uniform $L_{A_{e q}}$ time intervals.

In the second stage, measurements were made to elaborate the noise maps for two consecutive periods. The first was during August 1999 and the second during September and October 1999. A $200 \mathrm{~m}$ resolution square grid was drawn onto the town map (Navalmoral de la Mata has an approximate area of $1.7 \mathrm{~km}^{2}$ ). The sound level meter was placed at the nodes of this grid, or the nearest accessible points, with a total number of 59 measurement points. A similar grid size has been used in other studies. ${ }^{6,12,13}$ Fifteenminute measurements were made, within the time intervals established in the prior $L_{A_{e q}}$ stability study. These measurements will be described below. As an alternative to assigning a value to each square as an average of its corners, we elabo- 